\title{
Hören und Sehen
}

\section{Karl Rihaczek}

\section{Liebe Leserinnen, liebe Leser!}

Gestatten Sie mir, dass ich auf das einleitende Thema meines Editorials 09/06, „Die Gerechten und die Genauen“, zurückgreife. Dort sagte ich dem Sinne nach: In der biologischen Evolution setzt sich durch, was sich durchsetzt. Das kann das Starke, das Fruchtbare, das Anpassungsfähige, das vom (schöpferischen) Zufall Begünstigte etc. sein. Diese Reihe lässt sich nicht abschließend aufzählen. Was immer sich durchsetzt: Es setzt sich durch, was sich durchsetzt eine banale Erkenntnis.

Wir reden hier von biologischen Qualitäten. Ethische Qualitäten, also Tugenden wie Gerechtigkeit, Ordentlichkeit, Aufrichtigkeit etc. spielen hier allenfalls eine $\mathrm{Ne}$ benrolle. Das tugendhaft altruistische Individuum hat es gegen egoistische Konkurrenten schwer. Seine Tugenden machen auf die Evolution keinen unmittelbaren Eindruck. Anders die Gesellschaft, denn Tugenden ihrer Individuen bekommen einer Gesellschaft gut und heben ihre Bedeutung gegenüber Konkurrenten im Zuge der Evolution.

Biologische und moralische Qualitäten sind also verschiedener Art. Dennoch nennt man sie oft im gleichen Zuge. Etwa: König Artus war stark und gerecht. Da tut man eine biologische und eine moralische Qualität in den selben Sack und fragt sich, warum sich in der Evolution Gerechtigkeit nicht gleichermaßen augenfällig durchsetzt wie Stärke. Das liegt an dem genannten Unterschied. Dem Starken besorgt es die Biologie; für Gerechtigkeit haben wir zu sorgen. Mit Ethik hat die Evolution höchstens insofern zu tun, als sie uns für sie befähigt hat. Ausgedacht haben wir sie uns selber.

Ausgedacht haben wir uns auch die Technik. Man sollte meinen, dass wir dabei auch unsere Ethik zur Geltung gebracht haben, und dass sich die Technik demzufolge nicht dazu hergibt, jemandem Schaden oder Unrecht anzutun, dass sie sicher ist und unsere soziale Ordnung respektiert. Das ist, wie viel beklagt wird, leider nicht so. Die Technik entwickelt sich nicht nach einem ethisch geprüften menschlichen Generalplan sondern nach unzählbaren konkurrierenden Plänen. Das heißt: Sie entwickelt sich evolutionär, auf eine ethisch wertfreie Effektivität und Mächtigkeit hin, nach dem Grundsatz: Was geht, das geht. Was immer möglich ist, das setzen wir um. Skrupel kommen uns erst, nachdem wir Anstoß an negativen Begleiterscheinungen genommen haben. Dann versuchen wir, das Anstößige zu beseitigen, und sagen uns leichthin: So etwas soll uns nicht wieder passieren.

So stellte man z.B. in den 70-ger-Jahren fest, dass die Datenverarbeitung - damals ein unbescholtenes, allseits bestauntes, junges Phänomen - unsere Interessen als Individuen verletzt. Man beschloss, sie zu entschärfen und zu korrigieren, hatte aber für so eine Korrektur keinen wirtschaftlichen Ansatz. So beließ man die Datenverarbeitung so, wie sie sich entwickelt hatte, und verbot generell die Verarbeitung personenbezogener Daten. Nur was man an personenbezogener Datenverarbeitung für gerechtfertigt hielt, blieb vom Verbot ausgenommen. Dem Gesetzgeber wurde von den Technikern bedeutet: Finger weg von der Datenverarbeitungstechnik; sie entwickelt sich nach eigenen Gesetzen; du kannst dich nur blamieren.

Trotzdem: Wer will schon blind ins Unglück laufen? Die Technik sollte sich nach menschlichem Empfinden nicht unkontrolliert entwickeln. Man wollte deshalb seinerzeit die Technikfolgen abschätzen, um gegebenenfalls rechtzeitig korrigieren $\mathrm{zu}$ können. Das brachte einen Aufbruch. Im Kommunikationswesen, das zudem dereguliert werden musste, machte man sich $\mathrm{Ge}$ danken über zukünftige sozialverträgliche Dienstleistungen. Doch hat nichts Nennenswertes davon überlebt. Wir haben heute statt dessen das Internet, von dem damals keine Rede gewesen war. Die Technikentwicklung hatte sich beim besten Willen der besten Fachleute nicht abschätzen lassen. Wie hätte man dann deren soziale Folgen voraussehen können? Bleiben wir also dabei: Menschen entwickeln zwar die Technik, aber sie tun dies nicht nach einem Generalplan, sondern konkurrierend, ganz nach dem Muster der Evolution. Diese lässt der Menschheit keine andere Wahl.

Dennoch: Die Informationstechnik hält uns eine Eingriffsmöglichkeit offen: Sie bietet Programmierbarkeit. Damit können wir grundsätzlich die Technik auf Zwecke hin lenken, die unseren ethischen Werten aufgeschlossen sind. Wir können mit ihr unsere Umwelt organisieren, sie mit Datenverarbeitung sättigen und sicher machen. Mit der Mustererkennung (pattern recognition), RFID, Vernetzung, ubiquitärem Computing und der Möglichkeit, immer größere Datenmassen $\mathrm{zu}$ bewältigen kann diese Technik nicht nur programmiert entscheiden sondern auch selbst erkennen. Sie braucht uns zur Vorgabe der ethischen Bedingungen, aber nicht mehr als biomechanisches Zwischenglied. Sie schafft ihre Aufgaben wie z.B. das GPS General Positioning System - bis in eine unglaubliche Detailtiefe. Alles, was Struktur aufweist, kann sie an seinen individuellen Zügen erkennen, optisch wie akustisch. War die Informationstechnik bislang wohl imponierend aber blind und taub, so kann sie nun hören und sehen. Mein Gott, sind das Zeiten! Mit dieser sinnen- und detailbegabten Technik haben wir erstmals etwas an der Hand, mit dem wir unsere Tugenden in Technik umsetzen können.

Es fehlt allerdings noch, liebe Leserinnen und Leser, dass wir es wirklich tun. Man tut so einiges: Man kann per Mustererkennung feindliche Flugzeuge und Panzer von freundlichen unterscheiden und beruhigt schießen; man kann elektronische Steckbriefe ausstellen und den Gesuchten computerunterstützt optisch erkennen; man kann den individuellen Reiseverkehr auf der Welt mitsamt den Reisenden kontrollieren. Darüber hinaus liegt noch viel bislang ungenutztes Potenzial vor. Ob wohl eines Tages auch der Datenschutz davon profitiert und sich per Evolution ein wenig durchsetzt?

Mit freundlichen Grüßen, Ihr

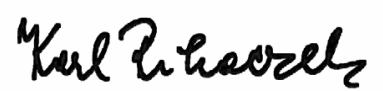

